\title{
A Comparative Study between Via-Hole and Via-Free Grounded Coplanar Waveguide to Microstrip Transitions on Thin Polymer Substrate
}

\author{
Mohammed El Gibari and HongWu Li \\ IETR Site de Nantes, Université de Nantes Angers Le Mans (UNAM), UMR CNRS 6164, 2 rue de la Houssinière, \\ BP 92208, 44322 Nantes Cedex 3, France
}

Correspondence should be addressed to Mohammed El Gibari; mohammed.el-gibari@univ-nantes.fr

Received 27 August 2015; Revised 3 November 2015; Accepted 6 December 2015

Academic Editor: Shih Yuan Chen

Copyright (C) 2015 M. El Gibari and H. Li. This is an open access article distributed under the Creative Commons Attribution License, which permits unrestricted use, distribution, and reproduction in any medium, provided the original work is properly cited.

\begin{abstract}
A comparative study between via-holed and via-free back-to-back GCPW-MS-GCPW (Grounded Coplanar Waveguide-Microstrip lines) transitions is reported in this paper. According to simulation results, both via-holed and via-free transitions on commercial benzocyclobutene polymer $20 \mu \mathrm{m}$ film show a bandwidth over $57 \mathrm{GHz}$. Bandwidth of optimized via-holed transitions increases with the via-hole diameter, up to $75 \mathrm{GHz}$ with $300 \mu \mathrm{m}$ via-hole diameter. The via-hole free transition achieves experimentally an ultrabroadband from $2 \mathrm{GHz}$ to $78 \mathrm{GHz}$ with an insertion loss of only $0.5 \mathrm{~dB}$ thanks to the copper metallization thickness of $2 \mu \mathrm{m}$. In addition, these measurement results are in perfect agreement with the simulation results. These via-free and via-holes transitions are very useful and requested in component packaging, on-wafer measurements of microstrip based microwave integrated circuits, and also the interconnections in hybrid circuits including both microstrip and coplanar structures.
\end{abstract}

\section{Introduction}

Telecommunications evolution is accompanied in particular for RF systems by a rise in frequency of operation and, for the optoelectronic systems, by an increase in the flows of information [1]. This evolution generates new requirements on components and functions for signal processing, especially constraints of integration. In order to ensure a good transmission quality, signal integrity must be preserved throughout the transmission. Different transmission lines can be used in circuits according to their topologies and functionalities. Transitions are then necessary to interface different transmission lines. High-performance microwave circuits require low losses and reflections at transitions interfaces, over large bandwidth for multiband or wideband applications. For example, electrooptic (EO) modulator is one of the key elements in optical transmission chains for very high bit-rate. Its microwave interconnections and driving circuits should be taken into account in order to achieve the maximum bandwidth. In this component based on EO polymer, microstrip line is suitable to apply the driving signal to optimize the EO modulation. Before packaging the final component, it is necessary to characterize its performances using a microwave probe station usually equipped with Ground Signal Ground (GSG) coplanar probes insuring a good electrical contact. Therefore, a transition between coplanar and microstrip lines (CPW-MS) is indispensable to characterize the components. This transition must satisfy at least these three criteria: ultrawideband, easy realization, and low cost.

To realize such transitions, the direct solution consists in physically connecting the top coplanar ground planes and the bottom ground plane of the microstrip line through metalized via-holes [2-4]. Although this solution is commonly used for thick layers, its use is very limited for thin layers. Indeed, as we will see later, the metalized via-holes degrade the pass-band flatness by giving rise to resonance peaks, especially in the case where the placement and the number of these via-holes are not optimized. Moreover, this technique is complex to realize, especially in the case 
of polymer modulators in which the microwave dielectric materials are constituted by optical and EO polymers which are very sensitive to photolithography chemistry. Via-free transitions are then an attractive solution [5-7] since they use capacitive coupling between the bottom and top ground planes to insure the ground continuity and do not require any processing on the substrates, but they are leading to a bandpass behavior cutting DC component. Via-free transitions on thin substrates, such as polymers films, can result in ultrabroad bandwidths due to a very large capacitance between the bottom and top ground planes. We reported on via-free GCPW (Grounded Coplanar Waveguide) to MS transitions on thin benzocyclobutene $(\mathrm{BCB})$ polymer substrate with a bandwidth up to $80 \mathrm{GHz}$ [8].

We report hereby on a comprehensive study of transitions between coplanar and microstrip lines on thin polymer films. The first section is devoted to a comparative study between via-hole and via-free transitions. We focus on the transition performance with optimized via-holes. This section aims to study the influence of the via-holes number and their diameter. In the second section, we present a theoretical study and an experimental validation of viafree back-to-back GCPW-MS-GCPW transitions with an ultrawide bandwidth without patterning the bottom ground plane. These transitions realized on thin BCB polymer film present an experimental bandwidth from $2 \mathrm{GHz}$ to $80 \mathrm{GHz}$. These via-free and via-holes transitions may be useful for all microwave components driven by microstrip lines, of which EO modulators based on polymer are only an example.

\section{GCPW-MS-GCPW Transitions}

The topology of GCPW-MS-GCPW transitions, with and without via-holes, studied in this paper is shown in Figure 1 where the microstrip line in between acts as driving electrode for functional device. The dielectric is a $20 \mu \mathrm{m}$ thick BCB polymer film that will stand for the stack of polymer layers realizing the EO waveguide. Its permittivity $\left(\varepsilon_{r}\right)$ is 2.65 and its loss tangent $(\tan \delta)$ varies from 0.008 to 0.02 depending on frequency $[9,10]$. The BCB polymer is deposited by spincoating on a $365 \mu \mathrm{m}$ thick silicon wafer which was previously full-wafer coated with a metallic film. The dimensions of the $50 \Omega$ microstrip and grounded coplanar lines are as follows: the width of the central strip $W=54 \mu \mathrm{m}$ and the coplanar gap $G=25 \mu \mathrm{m}$. The length of the microstrip line $L$ is fixed at $1 \mathrm{~cm}$. The deposited copper thickness is $2 \mu \mathrm{m}$ according to measurement with a profilometer. For these studies, we fixed the coplanar pads dimensions both $L_{1}$ and $S$ at $1 \mathrm{~mm}$. This choice is dictated by the study already done for these parameters [8].

In the grounded coplanar line structures, the electric field propagation spreads into three modes due to the backside conductor (cf. Figure 2(a)): microstrip (MS) mode, coplanar waveguide (CPW) mode, and coplanar microstrip (CPM) mode. In particular, the CPM mode is excited between the bottom ground plane and the coplanar ground strips on the top (it obviously does not exist without backside conductor in CPW configuration). The CPM mode could be avoided by optimizing the via-holes that prevent formation of a cavity
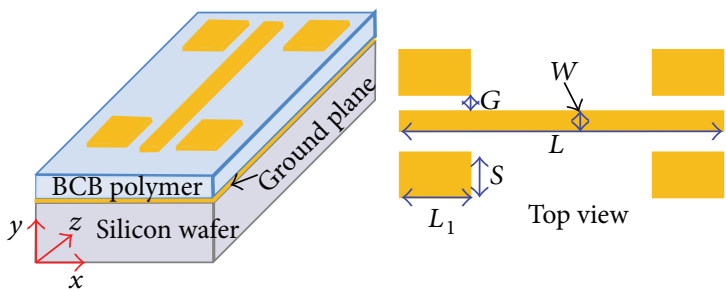

FIGURE 1: The studied back-to-back GCPW-MS-GCPW transitions with and without via-holes $\left(W=54 \mu \mathrm{m}, G=25 \mu \mathrm{m}, L=1 \mathrm{~cm}, L_{1}=\right.$ $1 \mathrm{~mm}$, and $S=1 \mathrm{~mm}$ ).

by the two lateral ground planes (cf. Figure 2(b)). These viaholes should indeed behave like a filter for the CPM mode.

\subsection{Comparison of the Back-to-Back GCPW-MS Transition} with and without Via-Holes. The transition presented in Figure 1 could have been realized using via-holes allowing a physical connection between the top coplanar ground planes and the bottom ground plane of the microstrip line. However, this technique increases the device manufacturing cost due to additional fabrication steps and requires special attention to the choice of the via-holes location, their diameter, and spacing between them. For this purpose several studies converge on the need to put more via-holes to effectively filter the CPM mode. Thus, as reported in $[11,12]$, the transmission properties of the transition can be improved by optimizing both the number of via-holes and their placement regarding the gap of the coplanar lines. Indeed via-holes placed along the coplanar line ( $z$-direction) and separated from one another by distance more than one half of the guided wavelength of the highest frequency of the band should eliminate or reduce the resonance peaks [12]. Recent studies have shown that the positions of these via-holes must be optimized in both $x$ - and $z$-directions to effectively reduce resonances peaks $[3,13]$. However, too many viaholes give rise to an increase of parasitic inductance which degrades the transmission performance [3]. These properties are well verified and validated with transitions on thick and high- $k$ substrate layers; so we focus on the performances of the transitions on low- $k$ thin substrate layers. We followed the explicit recommendations made by Zhou and Melde [3], concerning the metallized holes in $x$ - and $z$-directions (cf. Figure 3) to calculate the $S$-parameters of back-to-back GCPW-MS-GCPW transitions on thin BCB polymer film. The upper cutoff frequency of the dominant mode in GCPW line is given by the following relation:

$$
f_{c}=\frac{c}{2 \sqrt{\varepsilon_{r}}(d x-d)}
$$

where $c$ is the velocity of light in vacuum, $d x$ is the distance between two via-holes placed on both sides of the center conductor (cf. Figure 3), $d$ is the via-hole diameter, and $\varepsilon_{r}$ is the dielectric constant of the polymer. Therefore, the distance $d x$ must be as close as possible to the via-hole diameter $d$ but it should remain over the value $W+2 G+d$ [12]. Hence, with $W=54 \mu \mathrm{m}$ and $G=25 \mu \mathrm{m}$, in order to obtain $50 \Omega$ 


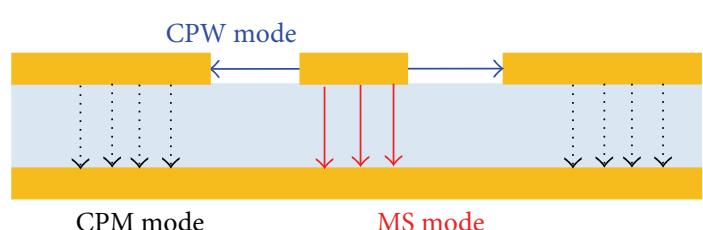

(a)

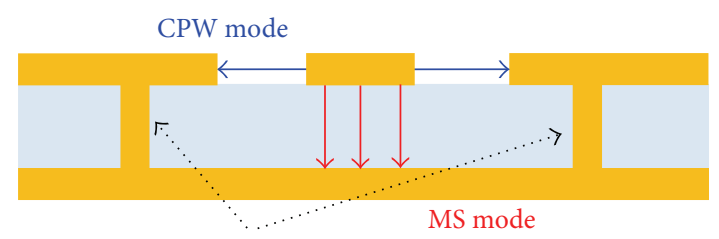

Via-hole

(b)

FIGURE 2: Different propagation modes in a grounded coplanar line structure; (a) without via-hole and (b) with via-holes.

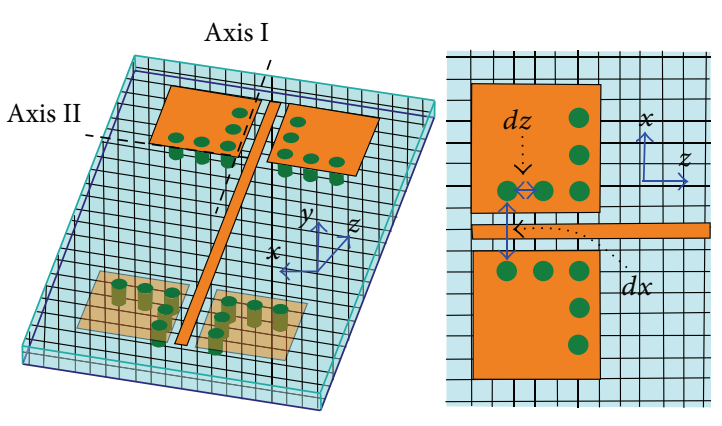

FIGURE 3: GCPW-MS-GCPW transition with optimized via-holes: $d x=d z=250 \mu \mathrm{m}$, the via-hole near the corner of the coplanar pads is located $200 \mu \mathrm{m}$ from both axes I and II, and via-hole diameter has 3 different values: 50,75 , and $100 \mu \mathrm{m}$ (not to scale).

characteristic impedance for both coplanar and microstrip sections, $d x$ is fixed to $250 \mu \mathrm{m}$, so it is much larger than $(W+2 G)$ and small enough to permit a reasonable number of via-holes over the coplanar pads. Secondly, as the bandwidth is much less sensitive to $d z$ (distance between two via-holes in $z$-direction) than to the distance $d x$ [3], we fixed also $d z$ to $250 \mu \mathrm{m}$. Three values, $100 \mu \mathrm{m}, 75 \mu \mathrm{m}$, and $50 \mu \mathrm{m}$, have been chosen for the via-hole diameter $d$, in order to figure out the influence of the via-hole diameter on results. However, relation (1) predicted an upper cutoff frequency of several hundred GHz. For example, if we take $d x=250 \mu \mathrm{m}, d=$ $50 \mu \mathrm{m}$, and $\varepsilon_{r}=2.65$, the upper cutoff frequency for the dominant mode should be $460 \mathrm{GHz}$.

The HFSS simulations results reveal some pieces of interesting information to consider for different structures in thin films. Therefore, in Figure 4 we present the results obtained for transitions with a few optimized via-holes, transitions with only one via-hole, and via-free transitions. DC component is naturally transmitted by all transitions with via-holes. But shallow resonant peaks begin to appear at lower frequencies than that with the via-hole free transition. It can be seen that resonance peaks frequency increases with increasing via-hole diameter. This is directly linked to the decrease of the via-hole inductance when diameter increases, which is a well-known phenomenon. Thus, resonance peaks are at $57 \mathrm{GHz}, 62 \mathrm{GHz}$, and $66 \mathrm{GHz}$ for via-hole diameters of, respectively, $50 \mu \mathrm{m}, 75 \mu \mathrm{m}$, and $100 \mu \mathrm{m}$. However, if we look at the performance obtained with only one optimized via-hole $(d=300 \mu \mathrm{m})$, we notice a very wide bandwidth (greater than $75 \mathrm{GHz}$ ). Indeed, to obtain this performance in thin layer, we need to reduce the via-hole inductance. This inductance can be estimated by formula (2) [14]. Consider

$$
L=\frac{\mu_{0}}{2 \pi}\left[h \cdot \ln \left(\frac{h+\sqrt{r^{2}+h^{2}}}{r}\right)+\frac{3}{2}\left(r-\sqrt{r^{2}+h^{2}}\right)\right],
$$

where $h$ is the dielectric thickness, $r$ is the via-hole radius, and $\mu_{0}\left(4 \cdot \pi \cdot 10^{-7} \mathrm{H} / \mathrm{m}\right)$ is the vacuum permeability. For dielectric thickness fixed, formula (2), supposed to be valid for dielectric thickness less than 3\% of free-space wavelength, shows that, to reduce the inductance via-hole, one must increase the via-hole diameter. Figure 5 presents HFSS simulation results of the transition with only one via-hole for different diameters $(50 \mu \mathrm{m}, 100 \mu \mathrm{m}, 200 \mu \mathrm{m}$, and $300 \mu \mathrm{m})$. We note clearly the influence of via-hole diameter on the resonance frequencies. In thin layer, one hole is sufficient for our GPCW-MS transition and the choice of via-hole diameter depends on the desired frequency band.

To understand the physical phenomenon underlying microwave properties of the studied transitions, we present in Figure 6 the evolution of the electrical field distribution at resonance peaks ( $57 \mathrm{GHz}, 62 \mathrm{GHz}$, and $66 \mathrm{GHz}$ ) for different via-holes diameters $(50 \mu \mathrm{m}, 75 \mu \mathrm{m}$, and $100 \mu \mathrm{m}$, resp.) in the top view of the GCPW-MS transition (cf. Figure 4). We observe that, whatever the via-holes diameter, we obtain the same electric field distribution. This shows that the resonance is simply the CPM mode caused by the coplanar ground plane surface [8]. If the via-holes diameters are large, the coplanar surface is reduced and therefore the CPM mode is excited at a higher frequency ( $66 \mathrm{GHz}$ for $100 \mu \mathrm{m}$ via-holes diameter) compared to structures where the diameter is small $(57 \mathrm{GHz}$ for $50 \mu \mathrm{m}$ via-holes diameter). The electrical field distribution in the case of the GCPW-MS transition with one via-hole (cf. Figure 7) shows that only one via-hole provides very good performance on the desired frequency band. We note that the propagating mode is a hybrid mode between the coplanar and the microstrip modes with a maximum of energy confined under the central strip thanks to the low thickness of the substrate.

The simulations results of the field distribution obtained with via-holes allow drawing two important conclusions: on the one hand, formula (1) is not appropriate for optimizing the number and placement of via-holes in low- $k$ films in thin layers in particular in the desired frequency band for less than $100 \mathrm{GHz}$ applications. On the other hand, a single optimized via-hole, in agreement with formula (2), gives a very wide 


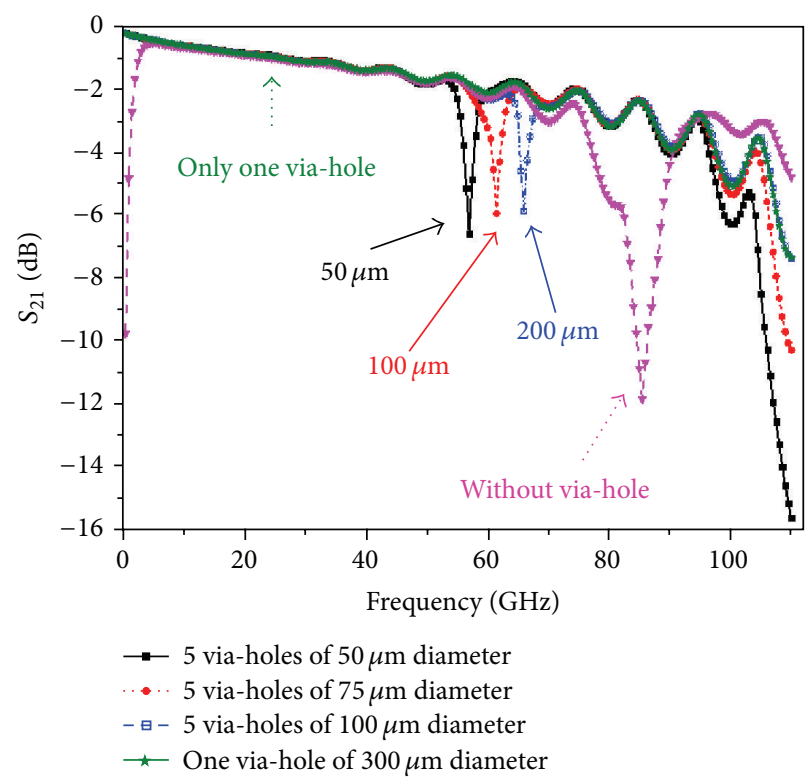

(a)

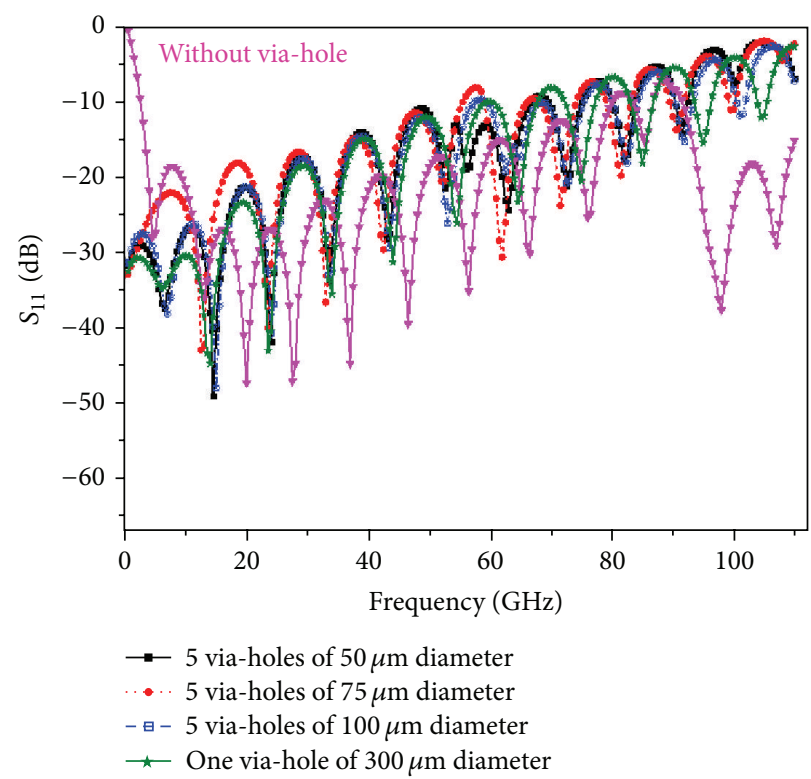

(b)

FiguRE 4: $S$-parameters of the back-to-back transitions without and with one optimized via-hole $(d=300 \mu \mathrm{m}$ located $500 \mu \mathrm{m}$ from both axes I and II; cf. Figure 3) and with five via-holes of different diameters $(50 \mu \mathrm{m}, 75 \mu \mathrm{m}$, and $100 \mu \mathrm{m})$ spaced by $250 \mu \mathrm{m}$ along $x$ - and $z$ - directions (cf. Figure 3). (a) Transmission coefficient $S_{21}(\mathrm{~dB})$ and (b) reflection coefficient $S_{11}(\mathrm{~dB})$.

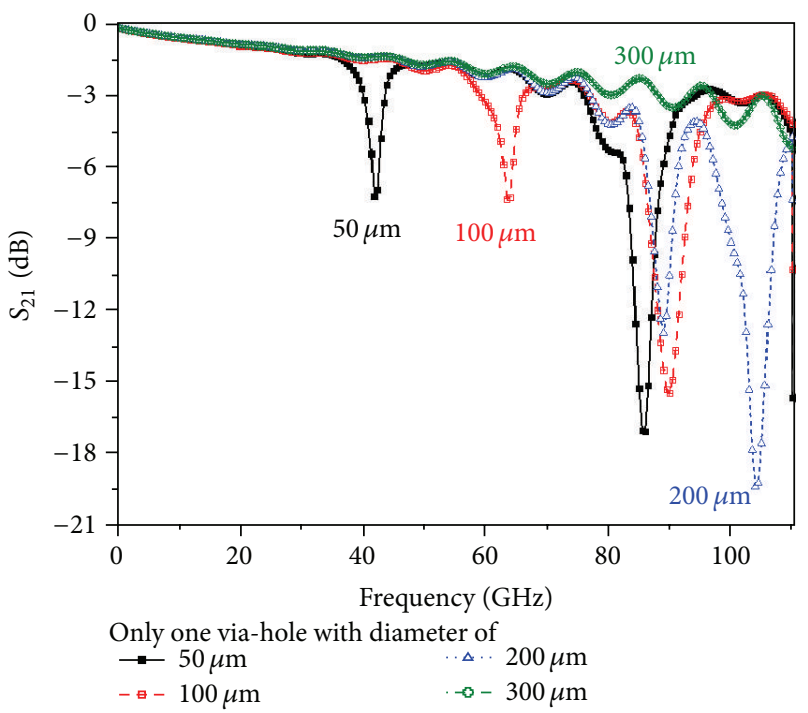

FIGURE 5: Transmission coefficient $S_{21}(\mathrm{~dB})$ of the back-to-back transitions with only one via-hole (located $500 \mu \mathrm{m}$ from both axes I and II; cf. Figure 3) for different diameters $(50 \mu \mathrm{m}, 100 \mu \mathrm{m}, 200 \mu \mathrm{m}$, and $300 \mu \mathrm{m})$.

bandwidth. This presents the advantage of having a large viahole diameter which facilitates the technical realization.

However, if we look at the results concerning the viafree transition, the first resonance peak takes place around $80 \mathrm{GHz}$, so via-free transitions have also an ultralarge bandwidth. Obviously, due to the capacitive coupling, low frequency range and DC do not go through the via-free transition. Indeed, the thickness and the permittivity of the dielectric substrate govern the bandwidth. The small thickness of the dielectric substrate has a double benefit: firstly, it can easily create the electromagnetic coupling between the bottom ground plane and top coplanar pads by capacitive effect; secondly, this small thickness facilitates the conversion of the CPW mode into the MS mode and pushes resonance peak to high frequency range [5]. In addition, the low- $k$ substrate is a very important factor to achieve broad bandwidth since parasitic resonances between bottom and top ground planes take place at higher frequencies owing to larger guided wavelengths for given frequencies [14]. Consequently, the GCPW-MS transition bandwidth relies on both the low- $k$ and the low thickness of the BCB polymer. It is noteworthy that the return loss parameter $\left(S_{11}\right)$ (see Figure 4(b)) got better values in the case of the via-free transition. As via-free transition can provide, at lower cost, higher upper cutoff frequency, the next paragraph is dedicated to the study of such via-free transitions. We provide a detailed explication to obtain a very large transition bandwidth depending on the desired applications. HFSS simulation results and measurements results up to $110 \mathrm{GHz}$ show a perfect agreement between them.

\subsection{Experimental Broadband Via-Free GCPW-MS-GCPW.}

Figure 8 presents the measurement and simulation results obtained with a transition realized on $20 \mu \mathrm{m}$ BCB polymer substrate, deposited by spin-coating on a $365 \mu \mathrm{m}$ thick metallized silicon wafer. For the upper metallization, this transition has a copper layer of $2 \mu \mathrm{m}$. The dimensions of the other parameters of the GCPW-MS-GCPW transition are as follows: $W=54 \mu \mathrm{m}, G=25 \mu \mathrm{m}, S=1 \mathrm{~mm}, L_{1}=1 \mathrm{~mm}$, and $L=1 \mathrm{~cm}$ (cf. Figure 1 for parameters definition). 


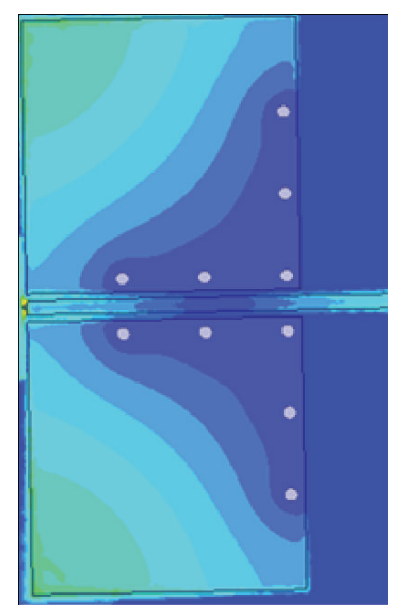

(a)
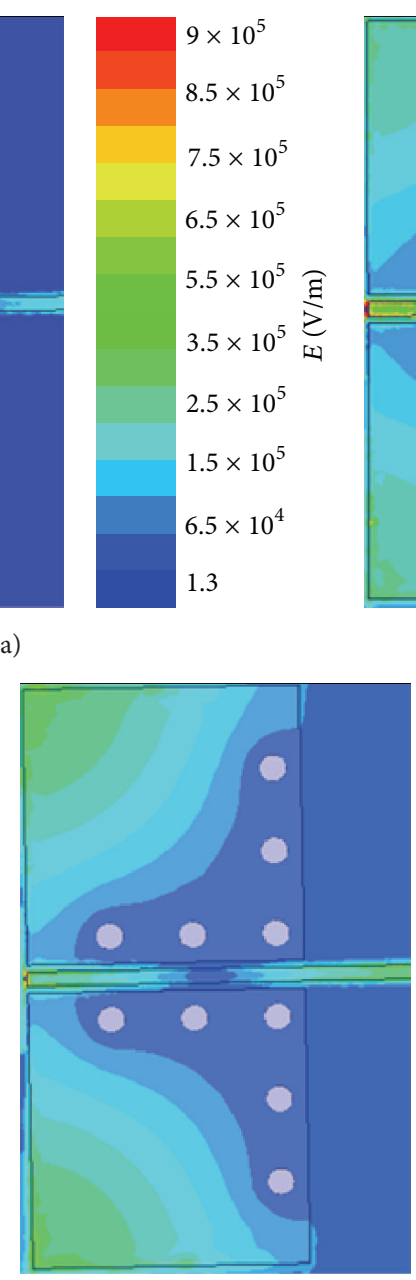
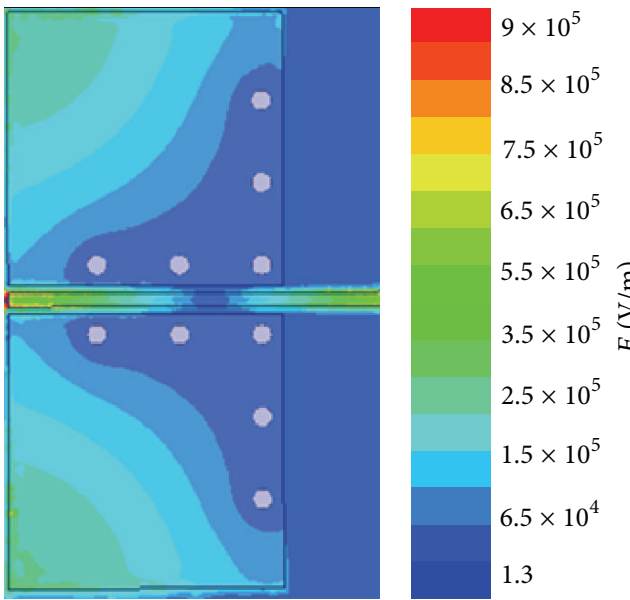

(b)

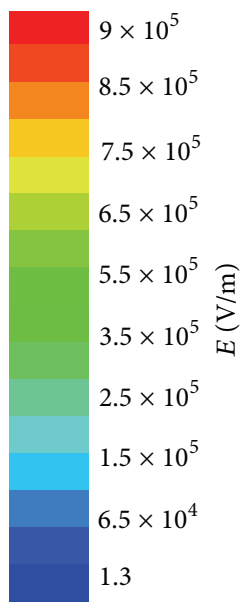

(c)

FIGURE 6: Electrical field distribution in a via-hole GCPW-MS transition at resonance peak; (a) via-hole diameter of 50 $\mu \mathrm{m}$ at 57 GHz, (b) via-hole diameter of $75 \mu \mathrm{m}$ at $62 \mathrm{GHz}$, and (c) via-hole diameter of $100 \mu \mathrm{m}$ at $66 \mathrm{GHz}$.

$S$-parameters have been measured using Agilent $8510 \mathrm{C}$ vector network analyzer whose bandwidth extends from $45 \mathrm{MHz}$ to $110 \mathrm{GHz}$ (single band) and Cascade illo-AGSG125 probes (110 GHz bandwidth, $125 \mu \mathrm{m}$ pitch). The calibration method used is the LRRM (Line-Reflect-Reflect-Match) method using a calibration substrate Cascade ISS-104-783. The whole system is controlled by Wincal Cascade software. All simulation results were obtained using the commercial 3D full-wave electromagnetic field analysis software HFSS version 15 . In order to carry out simulation in the conditions as close as possible to experimental procedure, coplanar probes were represented by the lumped port model with their exact parameters given in their datasheet. We note a very good agreement between experimental and simulation results. Due to the excellent electric continuity of the coplanar pads in $2 \mu \mathrm{m}$ thick copper, the transition performance is independent of the probe position on them according to both measurement and simulation results.

Considering that the upper and lower cutoff frequencies are defined with $3 \mathrm{~dB}$ insertion losses and $10 \mathrm{~dB}$ return losses, the measured bandwidth of the back-to-back GCPW-MSGCPW transition is from $2 \mathrm{GHz}$ to $78 \mathrm{GHz}$. The CPM mode causes the resonance peaks, at $86 \mathrm{GHz}$, in the $S_{21}$-parameter response curve by diverting input electromagnetic energy from the CPW mode.

Given the chosen copper metallization for its good conductivity, one can also improve the transition bandwidth on the BCB polymer by increasing copper metallization thickness. According to the transmission coefficient evolution of the GCPW-MS-GCPW transition with $4 \mu \mathrm{m}$ thick copper (solid line curve) in Figure 9, we could have a bandwidth of $85 \mathrm{GHz}$. Of course, the transition bandwidth can also be improved by using dielectric materials with both low permittivity and low loss tangent, such as the dielectric substrate CuFlon produced by Polyflon Company $\left(\varepsilon_{r}=2.1\right.$, $\tan \delta=0.00045)$. In Figure 9, we show the transmission coefficient results of this transition on the CuFlon substrate (dashed curve). Obviously, with the change of the substrate permittivity, the dimensions of the transition were adapted to keep a characteristic impedance of $50 \Omega$. So, with $20 \mu \mathrm{m}$ 


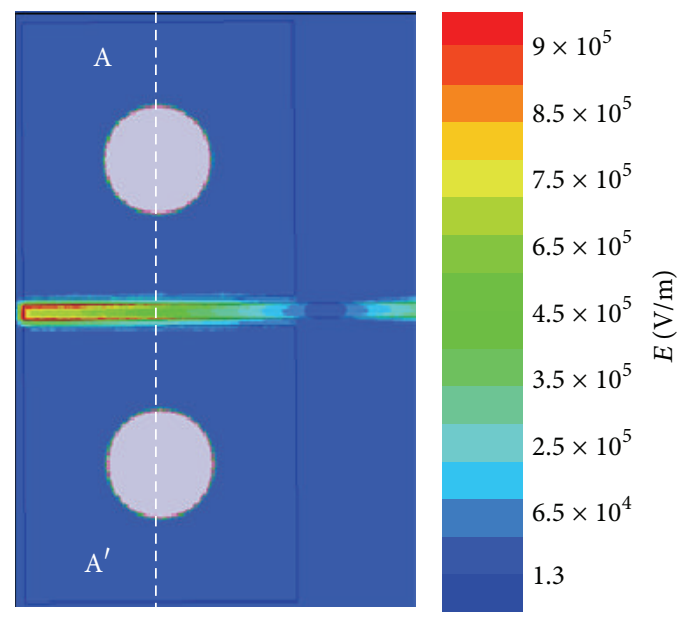

(a)
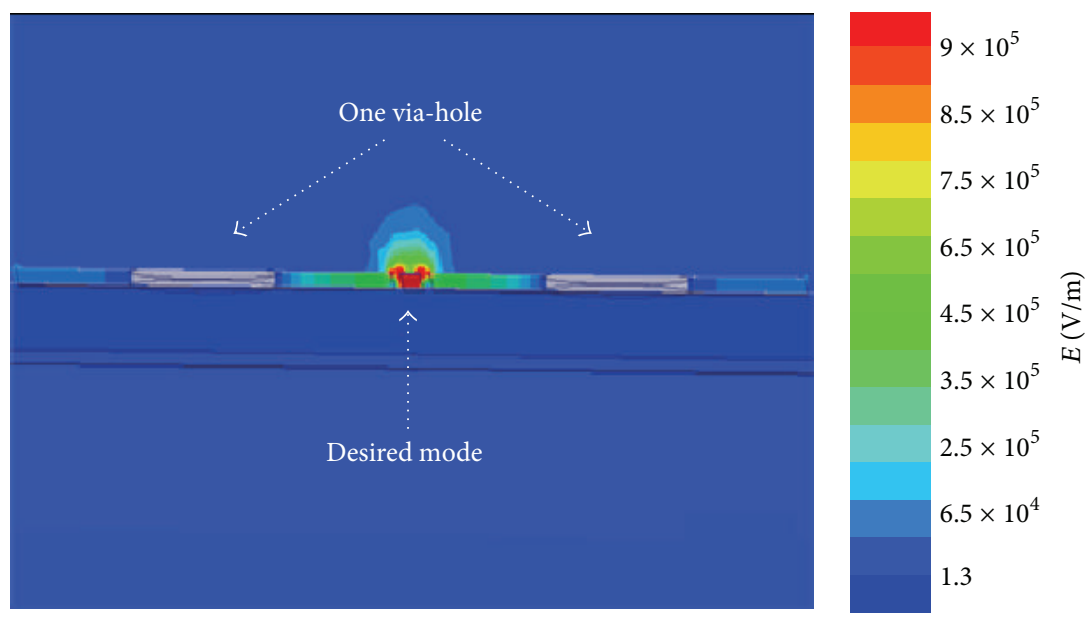

(b)

FIGURE 7: Electrical field distribution in the GCPW-MS transition with one via-hole at $40 \mathrm{GHz}(\mathrm{a})$ in the top view and (b) in the transverse section of the plane $\mathrm{A}-\mathrm{A}^{\prime}$.

CuFlon thickness, we took $W=61 \mu \mathrm{m}$ and $G=27 \mu \mathrm{m}$ and the thickness copper is fixed at $4 \mu \mathrm{m}$. In this case, we clearly observe the influence of the substrate permittivity on the bandwidth. The CPM mode takes place at $99.5 \mathrm{GHz}$ while it was excited at $88 \mathrm{GHz}$ in the same configuration with the $\mathrm{BCB}$ polymer. We also note that the transmission coefficient slope of the transition in CuFlon polymer is smaller than that of the transition in BCB polymer. This result shows the impact of the loss tangent on the bandwidth especially when the frequency increases. The transition realized with the CuFlon polymer could have a $92 \mathrm{GHz}$ bandwidth limited by the CPM mode excitation.

\section{Conclusion}

This paper has highlighted the impact of the metallized via-holes on the bandwidth of back-to-back GCPW-MS transitions in thin film. Via-holes ensuring physical contact between the coplanar ground planes and the bottom ground plane degrade the transition bandwidth, $60 \mathrm{GHz}$ for optimized via-holed transitions against optimized via-holes free transition on $20 \mu \mathrm{m}$ thick BCB polymer substrate and $2 \mu \mathrm{m}$ thick copper metallization. Via-free transitions are the optimal solution to achieve a broad bandwidth when DC component is not necessary. Indeed, thanks to thinness of the substrate, the electromagnetic coupling between the bottom ground plane and the coplanar pads is very large which facilitates field mode conversion without via-holes. Thus, we developed and analyzed back-to-back GCPW-MS-GCPW transitions with an experimental bandwidth of $78 \mathrm{GHz}$ and a low cutoff frequency of only $2 \mathrm{GHz}$. With such bandwidths and so high frequencies, equivalent circuits parameters are not easy to extract nor relevant due to the fact that the transition dimensions are larger than the guided microwave wavelength. In addition, perfect agreement between measurements results and HFSS simulations results was noted. These transitions, simple and inexpensive to build, will facilitate the characterization and the connectorization of components using microstrip driving electrodes. For monolithic microwave integrated circuit applications where space 


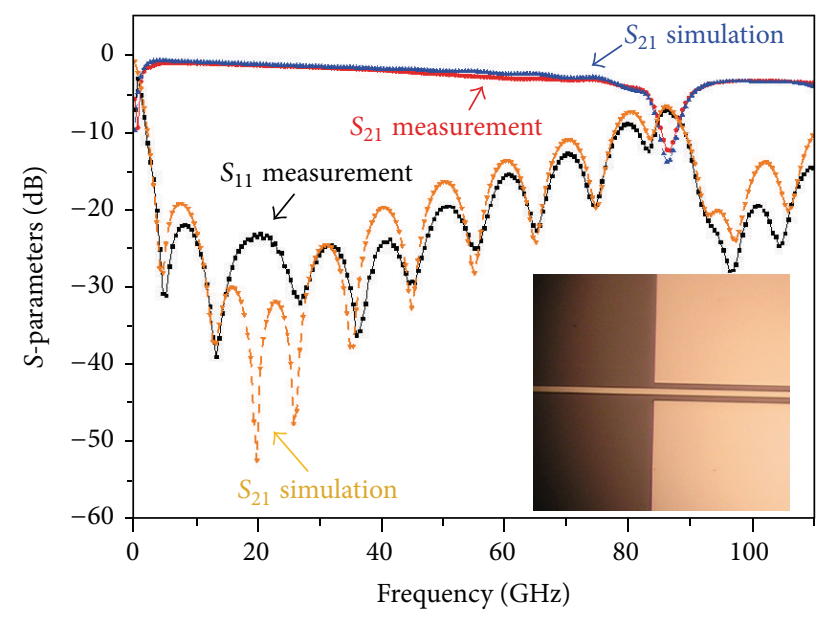

FIGURE 8: Measured and simulated $S$-parameters of via-free back-toback GCPW-MS-GCPW transition realized on $20 \mu \mathrm{m}$ BCB polymer substrate for $W=54 \mu \mathrm{m}, G=25 \mu \mathrm{m}, S=1 \mathrm{~mm}, L_{1}=1 \mathrm{~mm}, L=1 \mathrm{~cm}$, and $2 \mu \mathrm{m}$ thick copper metallization.

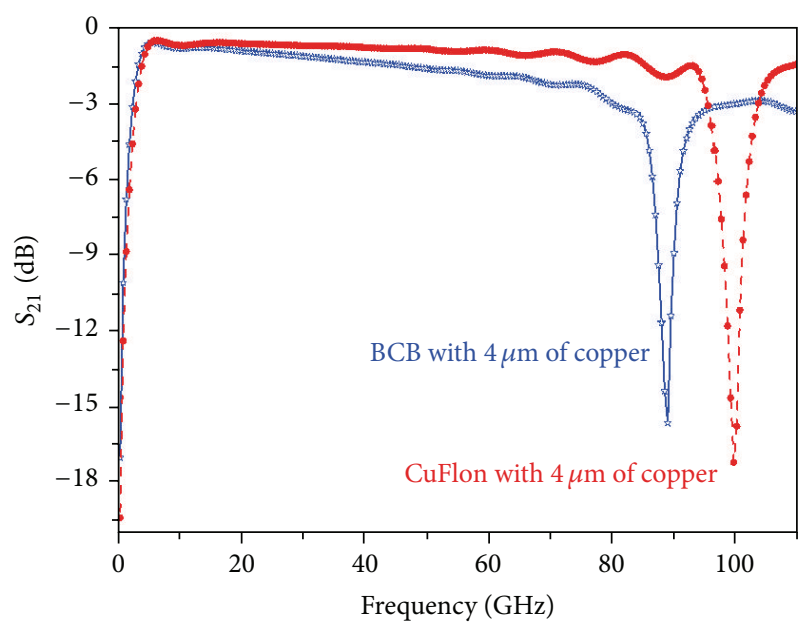

FIGURE 9: HFSS simulated $S_{21}$ transmission coefficient (dB) of viafree back-to-back transitions on polymer substrates CuFlon and $\mathrm{BCB}$, both of $20 \mu \mathrm{m}$ thickness.

is a critical element, it is possible to reduce coplanar pads dimensions to cover the desired bandwidth at the expense of low cutoff frequency. For example, with a coplanar pad of dimensions $S=L_{1}=200 \mu \mathrm{m}$, the bandwidth is greater than $90 \mathrm{GHz}$ with a lower cutoff frequency of $23 \mathrm{GHz}$ according to our calculation. We are developing realization method of metallized via-holes in the substrate, aiming at obtaining viaholes well defined in dimension and smoothly metallized. The technique is for the moment based on the cheapest chemical etching to make via-holes. Both chemical etching of crosslinked polymers and metallization of via-holes are difficult. Reactive ions etching and laser micromachining could be used to make via-holes but with a higher cost.

\section{Conflict of Interests}

The authors declare that there is no conflict of interests regarding the publication of this paper.

\section{References}

[1] A. Vilcot, B. Cabon, and J. Chazelas, Microwave Photonics from Components to Applications and Systems, Kluwer Academic Publishers, 2003.

[2] R. N. Simons, Coplanar Waveguide Circuits, Components and Systems, John Wiley \& Sons, 2001.

[3] Z. Zhou and K. L. Melde, "Development of a broadband coplanar waveguide-to-microstrip transition with vias," IEEE Transactions on Advanced Packaging, vol. 31, no. 4, pp. 861-872, 2008.

[4] B. K. O’Neil and J. L. Young, "Evaluation of coplanar waveguideto-microstrip transitions for precision S-parameter measurements," Microwave and Optical Technology Letters, vol. 50, no. 10 , pp. 2667-2671, 2008.

[5] L. Zhu and K. L. Melde, "On-wafer measurement of microstripbased circuits with a broadband vialess transition," IEEE Transactions on Advanced Packaging, vol. 29, no. 3, pp. 654-659, 2006.

[6] T. J. Ellis and J.-P. Raskin, "A broadband CPW-to-microstrip modes coupling technique," International Journal of Infrared and Millimeter Waves, vol. 23, no. 9, pp. 1357-1369, 2002.

[7] G. Zheng, J. Papapolymerou, and M. M. Tentzeris, "Wideband coplanar waveguide RF probe pad to microstrip transitions without via holes," IEEE Microwave and Wireless Components Letters, vol. 13, no. 12, pp. 544-546, 2003.

[8] M. El-Gibari, D. Averty, M. Halbwax, J. Vilcot, and H. Li, "Comprehensive study of ultra broadband GCPW-MS transition on thin films," Microwave and Optical Technology Letters, vol. 57, no. 9, pp. 2041-2045, 2015.

[9] M. D. Janezic, D. F. Williams, V. Blaschke, A. Karamcheti, and C. S. Chang, "Permittivity characterization of low-k thin films from transmission-line measurements," IEEE Transactions on Microwave Theory and Techniques, vol. 51, no. 1, pp. 132-136, 2003.

[10] J.-M. Kim, D. H. Oh, J.-H. Park et al., "Permittivity measurements up to $30 \mathrm{GHz}$ using micromachined probe," Journal of Micromechanics and Microengineering, vol. 15, no. 3, pp. 543$550,2005$.

[11] W. H. Haydl, "Resonance phenomena and power loss in conductor-backed coplanar structures," IEEE Microwave and Wireless Components Letters, vol. 10, no. 12, pp. 514-516, 2000.

[12] M. Yu, R. Vahldieck, and J. Huang, "Comparing coax launcher and wafer probe excitation for 10 mil conductor backed CPW with via holes and airbridges," in Proceedings of the IEEE MTT-S International Microwave Symposium Digest, vol. 2, pp. 705-708, IEEE, Atlanta, Ga, USA, June 1993.

[13] T. Kangasvieri, M. Komulainen, H. Jantunen, and J. Vähäkangas, "Low-loss and wideband package transitions for microwave and millimeter-wave MCMs," IEEE Transactions on Advanced Packaging, vol. 31, no. 1, pp. 170-181, 2008.

[14] M. E. Golfarb and R. A. Pucel, "Modeling via hole grounds in microstrip," IEEE Microwave and Guided Wave Letters, vol. 1, no. 6, pp. 135-137, 1991. 

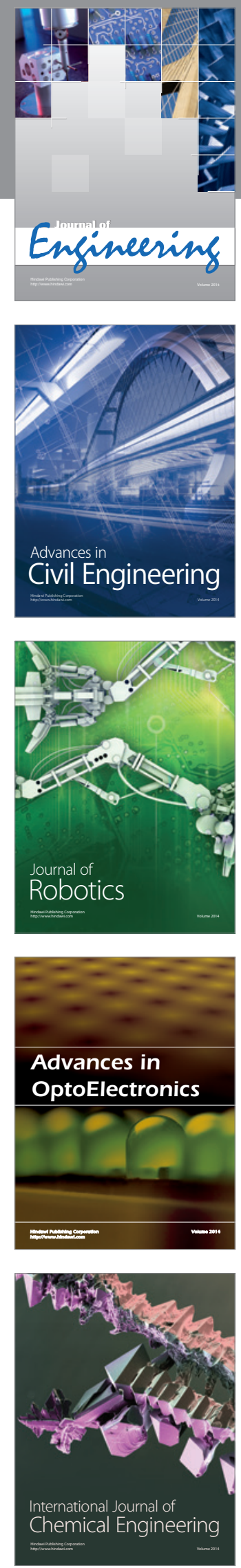

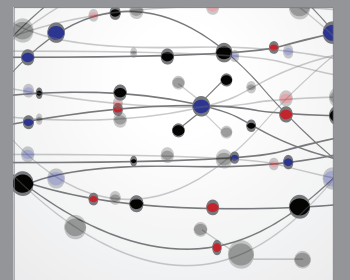

The Scientific World Journal
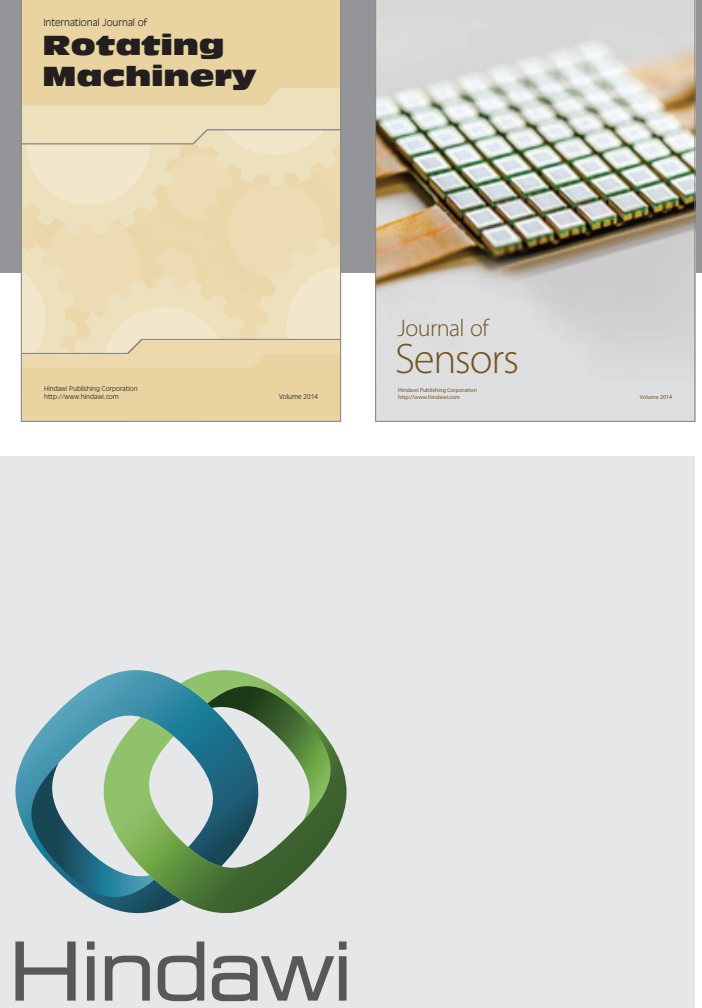

Submit your manuscripts at http://www.hindawi.com
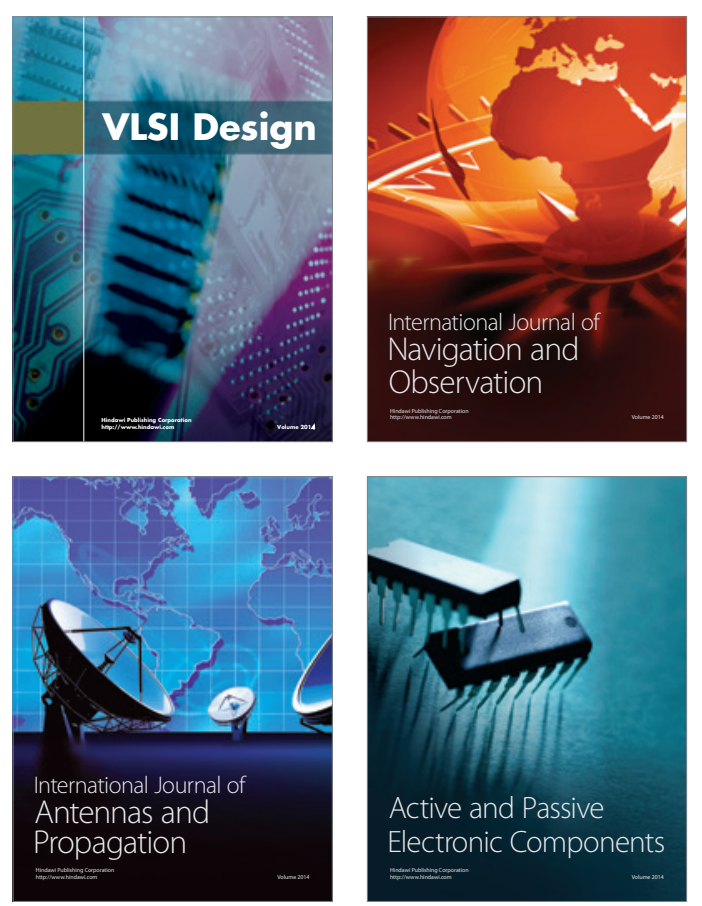
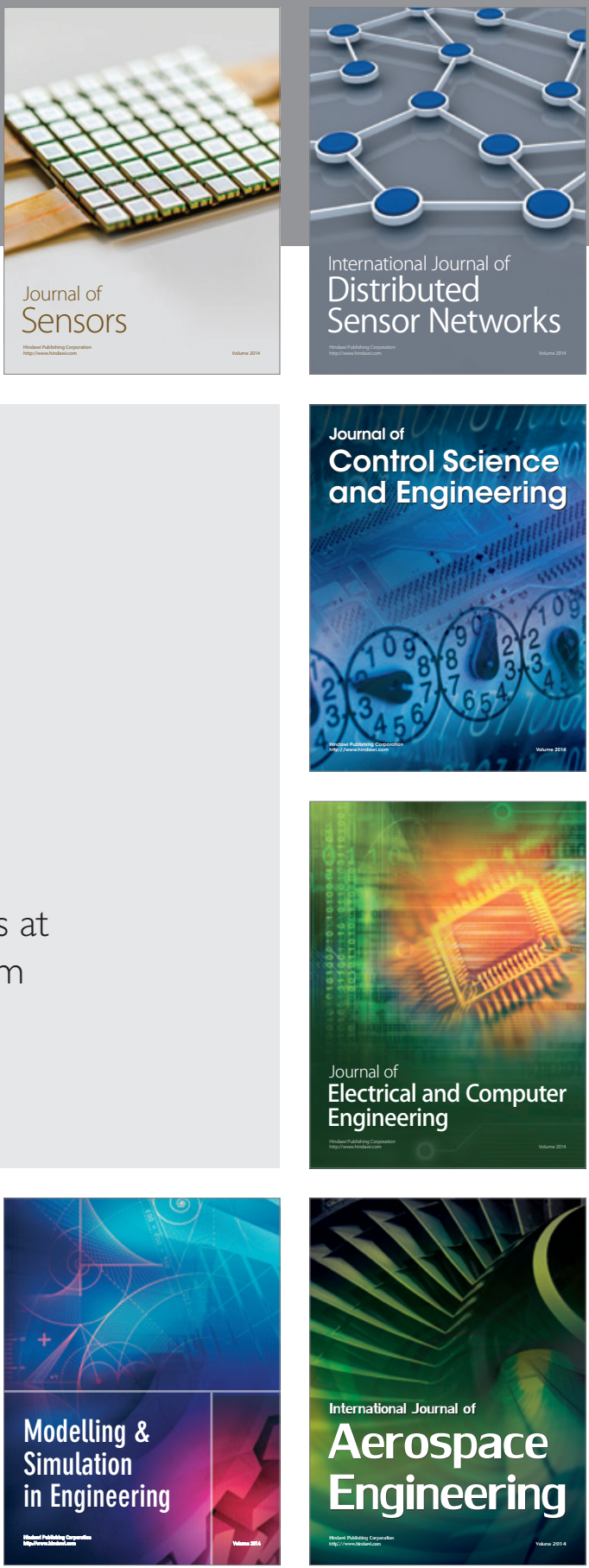

Journal of

Control Science

and Engineering
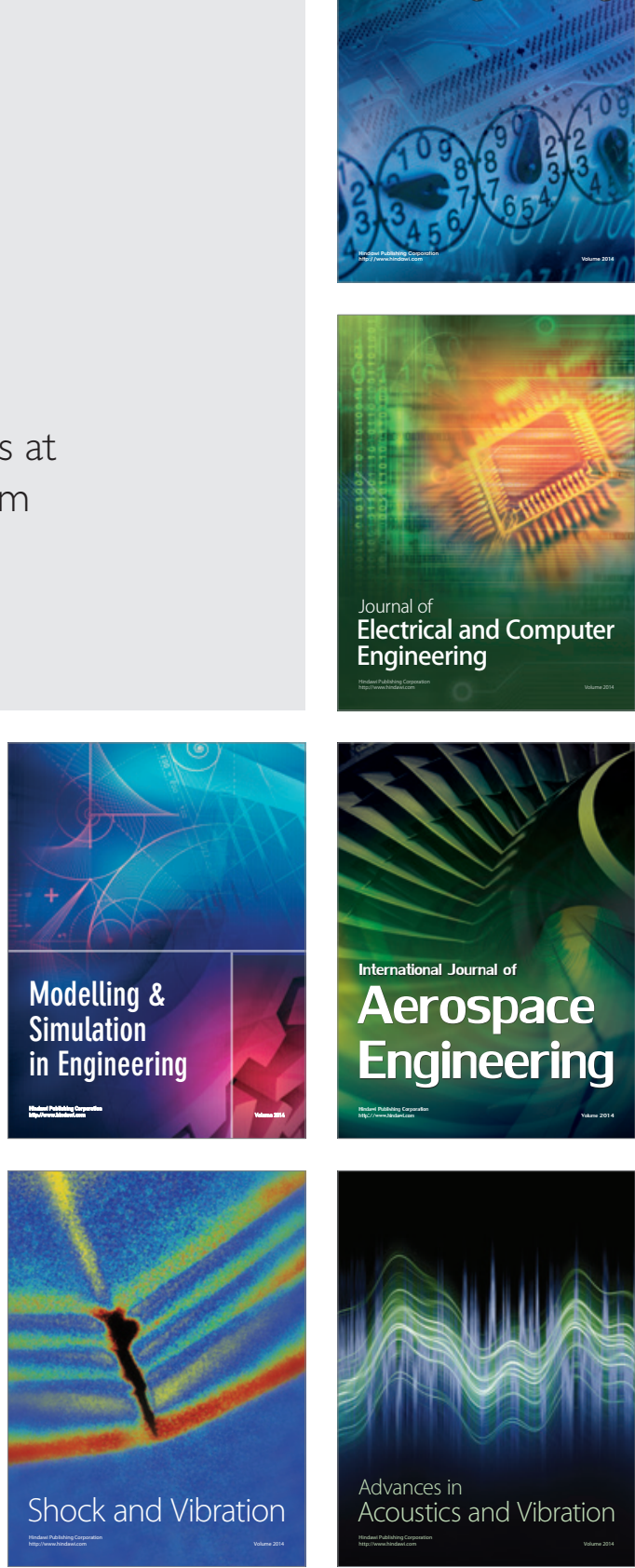